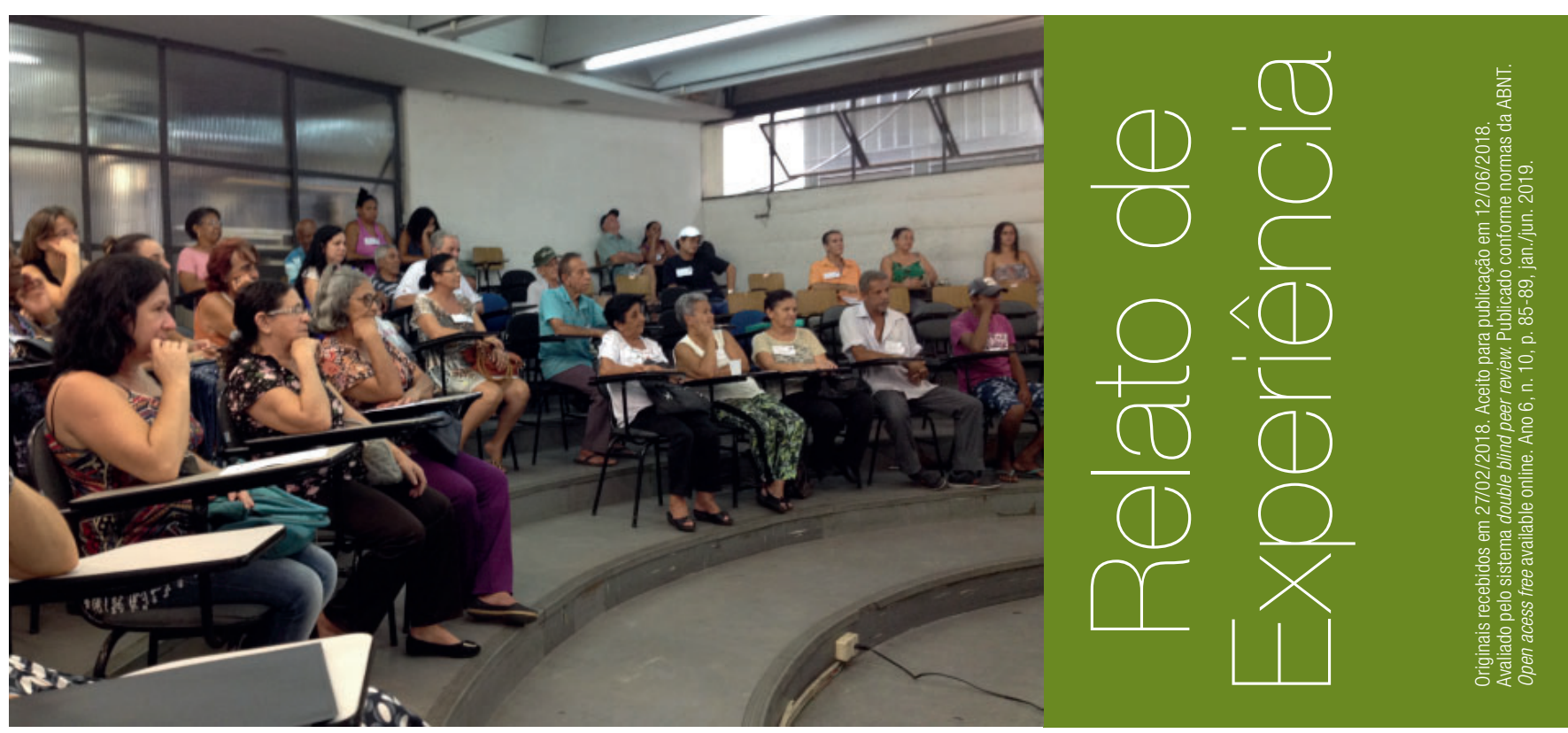

\title{
Atenção à saúde da pessoa com estomias em um programa de extensão universitária
}

Juliano Teixeira Moraes - julianotmoraes@ufsj.edu.br ${ }^{1}$

Rafaela das Graças Santiago Faria - rafaelafaria01@hotmail.com²

Deborah Franscielle da Fonseca - deborahfonseca@hotmail.com³

\section{RESUMO}

Este relato apresenta a experiência de um programa de extensão universitária que atua na promoção de saúde da pessoa com estomia. Dentre as atividades, o programa realizou visitas aos pacientes ainda no hospital, elaboração de uma cartilha educativa, orientações para 0 autocuidado e formação de grupos de apoio após a alta. 0 programa promoveu a articulação ensino-serviço e possibilitou a vivência da tríade ensino-pesquisa-extensão.

\section{PALAVRAS-CHAVE}

Educação em saúde. Enfermagem. Estomia. Autocuidado.

\footnotetext{
1 Doutor. Professor ajunto do curso de graduação em Enfermagem da Universidade Federal de São João del-Rei - Câmpus Centro Oeste.

2 Enfermeira. Universidade Federal de São João del-Rei - Câmpus Centro Oeste.

3 Enfermeira. Mestre em Enfermagem. Doutoranda em Ciências da Saúde. Universidade Federal de São João del-Rei - Câmpus Centro Oeste.
} 


\section{ABSTRACT}

This report presents experience of an university extension program in promoting the health of the person with the stoma. Among the activities, the program carried out visits to patients still in the hospital, preparation of an educational booklet, orientations for self-care and formation of support groups after hospital discharge. The program promoted the teaching-service articulation and made possible the experience of the teaching-research-extension triad.

\section{KEYWORDS}

Health education. Nursing. Stoma. Self-care.

\section{Relato de experiência}

A palavra "estoma" provém do grego e significa abertura de qualquer víscera oca através do corpo, podendo ser temporária ou definitiva, e nomeada de acordo com o órgão onde foi realizada a cirurgia (NASCIMENTO et al., 2011). Os estomas podem ser de alimentação, respiração ou eliminação. Sua confecção é um procedimento terapêutico, indicado como tratamento para neoplasias colorretais, diverticulite, doenças intestinais inflamatórias e traumas abdominais (MIRAND et al., 2014).

Os pacientes submetidos a cirurgia para confecção de uma estomia intestinal ou urinária enfrentam dificuldades relacionadas ao processo de aceitação e adaptação, que passa por estágios emocionais de ira, depressão, frustração, alteração da autoestima além de uma mudança significativa na qualidade de vida (COELHO; SANTOS; POGGETTO, 2013; MORAES; SOUSA; CARM0, 2012). Portanto é importante que o paciente pós-cirúrgico receba acompanhamento de reabilitação, pois neste período enfrentará diversas mudanças ao se deparar com um novo contexto de vida e situação de saúde (SANTANA, 2010).

Para lidar com essa demanda, algumas iniciativas têm sido desenvolvidas. No Brasil, os Serviços de Atenção à Saúde da Pessoa Ostomizada (SASPO) são unidades de saúde referência do Sistema Único de Saúde (SUS), responsáveis pela assistência e prestação de cuidados a estes pacientes (MORAES et al., 2014).

E, em nosso contexto, há o Programa Reabilitar de Atenção à Saúde da Pessoa Estomizada que apresentamos neste relato. É um programa de extensão da Universidade Federal de São João del-Rei - Câmpus Centro Oeste que tem por objetivo estimular o autocuidado e a reabilitação. Também busca contribuir para o desenvolvimento do serviço de saúde em rede do município, porque o momento da intervenção é principalmente entre o procedimento cirúrgico gerador de uma estomia e a inserção do estomizado no serviço. Na figura 1 pode-se observar os membros da equipe de trabalho

Desta maneira, o programa articula a universidade com a rede de assistência à saúde local. Além da instituição de ensino, fazem parte do programa a instituição hospitalar referência para a assistência no SUS e a atenção especializada (SASPO). Esta integração favorece a interação ensino-serviço-comunidade, contribuindo com a formação de profissionais qualificados.

Figura 1: Equipe de profissionais da saúde do Serviço Especializado, docente e alunos do Programa Reabilitar da UFSJ/CCO.

Fonte: Programa Reabilitar, 2016.

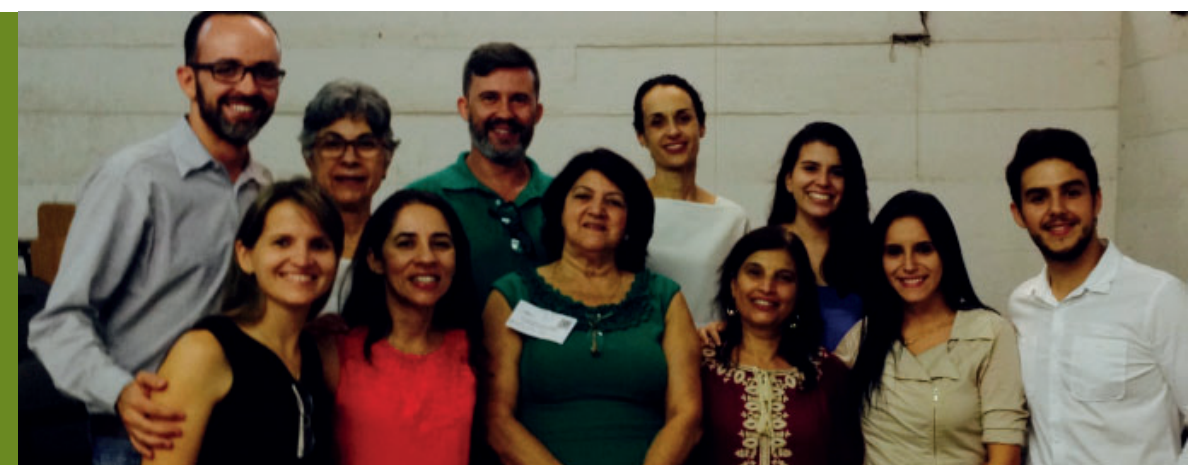


Destarte, o objetivo deste trabalho é relatar a experiência de acadêmicos dos cursos de Enfermagem e Medicina em um programa de extensão universitária que presta assistência aos pacientes recém-estomizados de um hospital de grande porte de Divinópolis, em Minas Gerais.

Para a operacionalização das atividades, os pacientes foram identificados por meio da visita hospitalar, realizada por alunos bolsistas e voluntários, e do encaminhamento realizado pela equipe de saúde do hospital diretamente à coordenação do programa. No período de abril de 2016 a março de 2017, foram assistidos 53 pacientes no pós-operatório de estomia (Tabela 1).

\begin{tabular}{|c|c|c|c|}
\hline Variável & númer & $\%$ & \multirow{16}{*}{$\begin{array}{l}\text { Tabela 1: Características sócio-clínicas } \\
\text { dos pacientes atendidos pelo Programa } \\
\text { Reabilitar, Divinópolis-MG, Brasil, 2016- } \\
2017 . \\
\text { Fonte: Programa Reabilitar, } 2016 .\end{array}$} \\
\hline \multicolumn{3}{|l|}{ Faixa etária } & \\
\hline$<20$ anos & 02 & 3,8 & \\
\hline 20-40 anos & 04 & 7,5 & \\
\hline 40-60 anos & 25 & 47,2 & \\
\hline$>60$ anos & 22 & 41,5 & \\
\hline \multicolumn{3}{|l|}{ Sexo } & \\
\hline Masculino & 31 & 58,5 & \\
\hline Feminino & 22 & 41,5 & \\
\hline \multicolumn{3}{|c|}{ Tipo de estoma } & \\
\hline Colostomia & 37 & 69.8 & \\
\hline lleostomia & 13 & 24,5 & \\
\hline Urostomia & 03 & 5,7 & \\
\hline \multicolumn{3}{|c|}{ Permanência do estoma } & \\
\hline Temporário & 24 & 45,3 & \\
\hline Definitivo & 29 & 54.7 & \\
\hline
\end{tabular}

As visitas hospitalares ocorreram três vezes por semana. Durante as visitas, foi observado que 83\% dos pacientes atendidos pelo Programa não tinham recebido nenhum tipo informação quanto ao procedimento de confecção do estoma antes da cirurgia. E que, ao se depararem com a estomia pela primeira vez, alguns pacientes se recusavam inclusive a vê-la.

Nesse momento, surgiam inúmeras dúvidas acerca dos cuidados com o dispositivo coletor e com a estomia, além daquelas que diziam respeito a como agir e o que fazer no seu cotidiano. Questionavam se algo mudaria na sua rotina diária, se seu desempenho sexual sofreria algum dano ou se existia alguma restrição alimentar ou laboral.

Para facilitar o processo de ensino-aprendizagem entre os envolvidos no programa e os pacientes, foi elaborada uma cartilha com orientações importantes para 0 autocuidado em estomias. Esta cartilha abordava cuidados básicos com higiene, alimentação e atividade física. Abordaram-se também questões emocionais, de sexualidade e ainda os direitos assegurados por lei para aquele grupo (Figura 2).

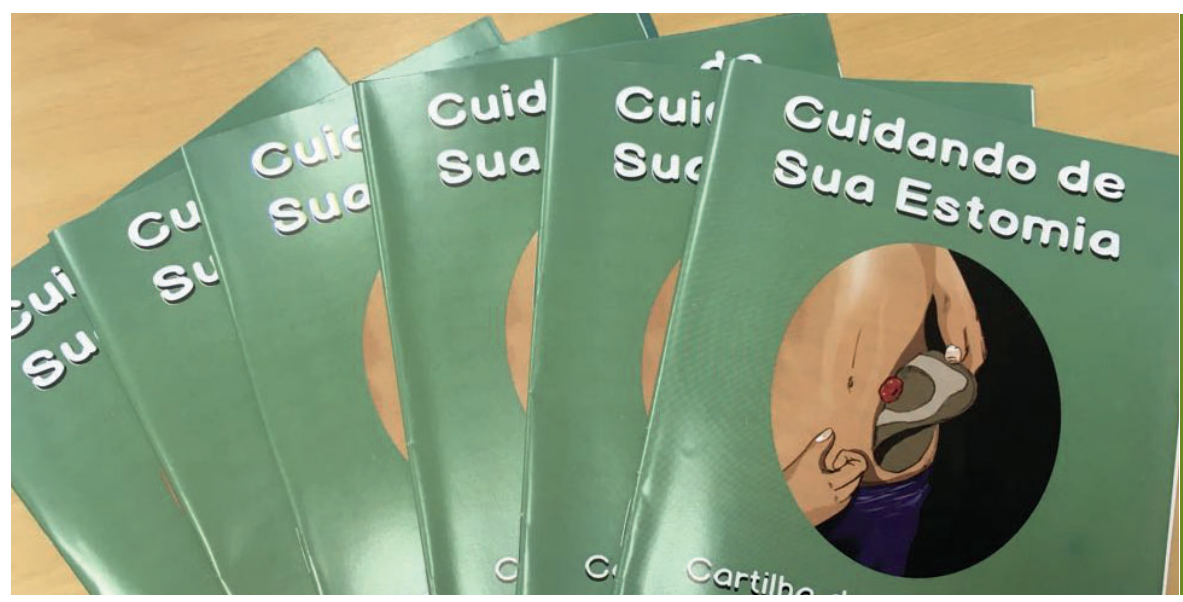

Figura 2: Cartilha de cuidados para a assistência à pessoa com estomias

Fonte: Programa Reabilitar, 2016. 
Então, durante a visita hospitalar, foram oferecidas orientações sobre 0 uso dos dispositivos, avaliação do estoma e entrega da cartilha. E então cada paciente foi encaminhado ao serviço público de referência de seu município de origem. 0 programa forneceu ainda um kit contendo quatro dispositivos coletores, um medidor, um guia de orientações, um espelho para auxiliar no autocuidado e um nécessaire para cada paciente.

No período pós-alta hospitalar, esses pacientes são acompanhados ambulatorialmente pelos SASPO. Para aquelas pessoas com estomias do município de Divinópolis-MG, o programa também desenvolveu ações neste espaço, dentre as quais citam-se 0 atendimento clínico interdisciplinar e os grupos de apoio.

Esses grupos de apoio permitem que os pacientes façam a troca de experiência com pessoas que vivem na mesma condição, contribuindo para o processo de reabilitação (SILVA, 2014). Nesses grupos, houve a participação das pessoas estomizadas em todas as fases da ação. Ali eles tinham a oportunidade de esclarecer dúvidas, conhecer dispositivos e adjuvantes, compartilhar experiências e também de fazer amizades com pessoas que compartilham da mesma situação. Assim, foi possível favorecer a troca de experiências entre eles, além de contribuir com conceitos e conteúdos que favorecem a qualidade de vida.

Essa inserção nas atividades do SUS pôde melhorar a abrangência do Programa Reabilitar, possibilitando uma articulação dos atendimentos realizados no hospital com o SASPO. Além disso, foi um facilitador da inserção dos indivíduos no contexto do SUS.

0 Programa Reabilitar alcançou sua proposta de intervenção junto à comunidade, uma vez que seus objetivos foram amplamente efetivados. A experiência contribuiu tanto para a formação profissional da equipe quanto para 0 desenvolvimento de habilidades técnicas e competências pessoais dos pacientes, por meio da capacitação e elucidação de dúvidas dos estomizados.

Em uma atividade de extensão, o estudante tem a oportunidade de usar seus conhecimentos adquiridos no meio acadêmico somados às suas habilidades culturais e sociais. Essa prática ainda permite devolver à sociedade 0 investimento público no ensino superior. Foi uma oportunidade de troca de saberes, por meio do diálogo, entre serviços, discentes e docentes da UFSJ-CCO, profissionais do hospital, da equipe multiprofissional do SASPO e pacientes. Assim, tornou-se possível oferecer à sociedade alguns dos conhecimentos acadêmicos em benefício do desenvolvimento da assistência à saúde local.

Entendemos que este trabalho foi expressivo para município, pacientes e principalmente para os integrantes do Programa Reabilitar de Atenção à Saúde da Pessoa Estomizada. Possibilitou a articulação teórico-prática, gerando pesquisa e consequentemente contribuindo para ampliação do conhecimento científico do futuro profissional, amadurecimento, aprimoramento, crescimento pessoal e construção da cidadania.

\section{Agradecimentos}

Agradecemos à equipe de profissionais do Serviço de Atenção à Saúde das Pessoas Estomizadas de Divinópolis - MG: Marlene das Dores Medeiros, Magda Eva da Silva, Nize Renê Ferreira e aos alunos bolsistas Isabele Elita de Oliveira Neves e Natanael Aguiar Airão.

\section{Referências}

COELHO, A. R.; SANTOS, F. S.; POGGETTO, M. T. D. A estomia mudando a vida: Enfrentar para viver. Revista Mineira de Enfermagem, Belo Horizonte, v. 17, n. 2, p. 238-267, abr/ jun. 2013.

MIRAND, S. M. et al. Viver com Estomia: Contribuições para a Assistência de Enfermagem.

Revista Estima, v. 12, n. 3. 2014. 
MORAES, J. T. et al. Serviços de atenção ao estomizado: análise diagnóstica no Estado de Minas Gerais, Brasil. Cadernos Saúde Coletiva, Rio de Janeiro, v. 22, n. 1, p. 101-108, jan./ mar. 2014

MORAES, J. T.; SOUSA, L. A.; CARMO, W. J. Análise do autocuidado das pessoas estomizadas em um município do centro oeste de Minas Gerais. R. Enferm. Cent. O. Min, Divinópolis, v.2, n. 3, p. 337-346, set/dez 2012.

NASCIMENTO, C. M. S et al. Vivência do paciente estomizado: uma contribuição para a assistência de enfermagem. Texto contexto - enferm. [online], v. 20, n. 3, p. 557-564, 2011.

SANTANA, J. C. B et al. 0 significado de ser colostomizado e participar de um programa de atendimento ao ostomizado. Cogitare Enferm. v. 15, n. 4, p. 631-8, out/dez, 2010.

SILVA, R. O. da. Estomia Intestinal: dificuldades na adaptação e no desenvolvimento do autocuidado (Revisão Integrativa da Literatura). Universidade de Brasília, Faculdade de Ciências da Saúde, Departamento de Enfermagem, Brasília - DF, 2014. 